\title{
Safety and Efficacy of Early High Parenteral Lipid Supplementation in Preterm Infants: A Systematic Review and Meta-Analysis
}

\author{
Kyunghoon $\mathrm{Kim}^{1}{ }^{\mathbb{D}}$, Na Jin $\mathrm{Kim}^{2}$ and Sae Yun Kim ${ }^{1, *(\mathbb{D})}$ \\ 1 Department of Pediatrics, College of Medicine, The Catholic University of Korea, Seoul 06591, Korea; \\ journey237@catholic.ac.kr \\ 2 Medical Library, The Catholic University of Korea, Seoul 06591, Korea; kimnj@catholic.ac.kr \\ * Correspondence: sysmile@gmail.com; Tel.: +82-10-3743-3600
}

check for updates

Citation: Kim, K.; Kim, N.J.; Kim, S.Y. Safety and Efficacy of Early High

Parenteral Lipid Supplementation in Preterm Infants: A Systematic Review and Meta-Analysis. Nutrients 2021, 13, 1535. https://doi.org/10.3390/ nu13051535

Academic Editor: Asim K. Duttaroy

Received: 7 April 2021

Accepted: 29 April 2021

Published: 2 May 2021

Publisher's Note: MDPI stays neutral with regard to jurisdictional claims in published maps and institutional affiliations.

Copyright: (c) 2021 by the authors. Licensee MDPI, Basel, Switzerland. This article is an open access article distributed under the terms and conditions of the Creative Commons Attribution (CC BY) license (https:// creativecommons.org/licenses/by/ $4.0 /)$.

\begin{abstract}
The objective of this systematic review and meta-analysis was to summarize the effects of early initiation and achievement of a high dose of parenteral lipids $(\geq 1.5 \mathrm{~g} / \mathrm{kg} /$ day reached within the first $24 \mathrm{~h}$ of birth) on growth and adverse outcomes in preterm infants. PubMed, EMBASE, and Cochrane databases were utilized to search for publications for this meta-analysis. Randomized controlled trials were eligible if data on growth or clinical outcome was available. The search returned nine studies. The mean proportion of postnatal weight loss (\%) was lower (mean difference [MD]: $-2.73 ; 95 \%$ confidence interval [CI]: $-3.69,-1.78)$, and the mean head circumference near the term equivalent age $(\mathrm{cm})$ was higher in the early high lipid treatment group (MD: 0.67; 95\% CI: 0.25, 1.09). There was a favorable association of early high lipid administration with the incidence of extrauterine growth restriction (relative risk [RR]: $0.27 ; 95 \%$ CI: $0.15,0.48$ ). Generally, there were no differences in morbidities or adverse outcomes with early high lipid administration. Early initiation of parenteral lipids and high dose achieved within the first $24 \mathrm{~h}$ of life appear to be safe and endurable and offer benefits in terms of growth.
\end{abstract}

Keywords: lipids; preterm infants; growth; adverse effect

\section{Introduction}

The goal of nutrition for preterm infants is to mimic the intrauterine growth rate and to achieve favorable long-term developmental outcomes. Despite recent enhanced nutritional support in neonatal medicine [1,2], achieving this weight gain goal is still very difficult, especially during the first week of life when preterm infants are exposed to highest risk of undernutrition. Insufficient nutrition during this critical period results in cumulative energy and protein deficits that aggravate the postnatal weight loss [3].

Lipids are indispensable components for preterm infants in the early stage of postnatal life because of the essential fatty acid supply for growth and development and their high energy density. Recent meta-analyses provided evidence that the early initiation of lipids in preterm infants appears to be safe and tolerable but that there are no significant benefits of lipid administration before $2-5$ days of age [4,5]. Additionally, a study in France reported that early introduction of parenteral lipids improved neonatal growth and that the lipid dose was positively associated with weight gain in very preterm infants [6] and possible later neurodevelopmental outcomes [7]. The latest recommendation suggested by the European Society for Pediatric Gastroenterology Hepatology and Nutrition (ESPGHAN) in 2018 showed that, in preterm infants, intravenous lipid emulsion (IVLE) can be started immediately after birth [8]. To date, "early" is not defined as an immediate time, but in a recently published randomized controlled study on the administration of IVLE in very preterm infants, researchers compared more immediate timing, within $6 \mathrm{~h}$ after birth versus between 12 and $24 \mathrm{~h}$ after birth, showing that the provision of a high amount of lipids early results in less weight loss and better head growth, in terms of the z-score [9]. 
In the aforementioned ESPGHAN guideline, for preterm infants, parenteral lipid intake should not exceed $4 \mathrm{~g} / \mathrm{kg} /$ day because of adverse effects such as lipid intolerance; however, there was no consensus about how high a dose of IVLE was endurable and optimal in the early postnatal period. Additionally, there was little evaluation of long-term efficacy and safety, which makes concrete recommendations difficult to be established. Consequently, the objective of this meta-analysis is to find out the short-term efficacy and safety of early high IVLE introduction to preterm infants; dose of IVLE is equal to $1.5 \mathrm{~g} / \mathrm{kg} /$ day or more within $24 \mathrm{~h}$ after birth. Anthropometric data and possible adverse effects associated with early high IVLE administration were investigated to determine the efficacy and safety.

\section{Materials and Methods}

\subsection{Search Strategy}

We performed a systematic review and meta-analysis in accordance with the preferred reporting items for systematic reviews and meta-analyses (PRISMA) guidelines [10]. We did a systematic search employing a protocol designed by an independent medical librarian (N.J. K) with three electronic databases: PubMed, EMBASE, and Cochrane Library. To qualify for inclusion, studies had to be randomized controlled trials (RCT) comparing interventions that differed only in the dose of parenteral lipids that were administered to preterm babies. We used the search terms listed in the supplemental file (S1) to search three electronic databases. We imposed no language or publication restrictions.

\subsection{Study Selection}

Two reviewers (K.K. and S.Y.K.) independently evaluated the titles and abstracts obtained from the search for the first screening. After the initial exclusion process, two reviewers (K.K. and S.Y.K.) independently reviewed the full texts of the remaining articles to determine whether any articles met the eligibility criteria: an RCT design; a study group of preterm infants weighing $<1500 \mathrm{~g}$ who were admitted to a neonatal intensive care unit (NICU), necessitating parenteral nutrition, and were administered any type of parenteral lipid emulsion within the first $24 \mathrm{~h}$ of life; and growth data included as an outcome measure. We resolved disagreement by discussion. We recorded the selection process in sufficient detail to complete the PRISMA flow diagram (Figure 1).

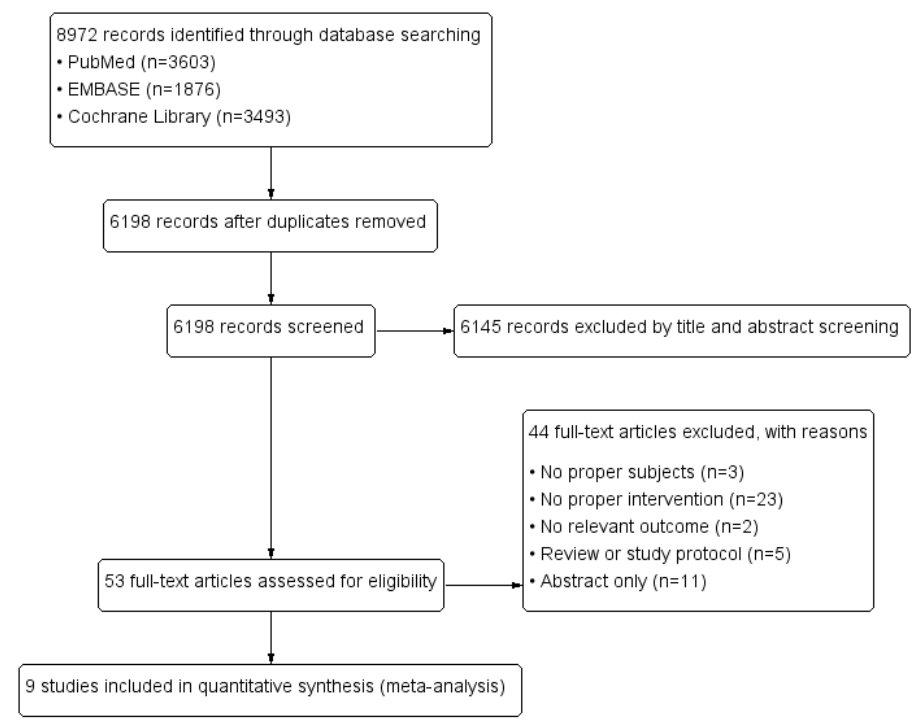

Figure 1. Overview of the selection process throughout the study.

\subsection{Data Extraction and Assessment of Risk of Bias}

Both reviewers (K.K. and S.Y.K.) used a structured format to extract data from each eligible study. Data could be categorized as characteristics of the sample, intervention 
details, and measurement of outcomes. We resolved disagreement by discussion. Outcome measures were divided into primary outcomes on growth divided into six components and ten secondary outcomes. Primary outcomes were as follows: (1) weight gain rate ( $\mathrm{g} / \mathrm{kg} /$ day); (2) proportion of postnatal weight loss (\%); (3) time to regain birth weight (BW) (d); (4) body weight near term equivalent age (TEA) (g); (5) head circumference near TEA $(\mathrm{cm})$; and (6) extrauterine growth restriction (EUGR) defined by weight less than 10th percentile for postmenstrual age (PMA) based on the Fenton growth chart [11]. Secondary outcomes were as follows: (1) death before NICU discharge; (2) length of NICU stay (d); (3) incidence of bronchopulmonary dysplasia (BPD) or chronic lung disease (CLD) defined as oxygen therapy; (4) incidence of necrotizing enterocolitis (NEC) stage $\geq 2$ on the modified Bell's staging criteria [12]; (5) incidence of culture proven sepsis; (6) severe intraventricular hemorrhage (IVH) with a Papile classification of grade 3 or 4 [13]; (7) retinopathy of prematurity (ROP), defined as any stage of ROP after birth detected by ophthalmoscopy, as defined by the International Classification of Retinopathy of Prematurity [14]; (8) incidence of hypertriglyceridemia and serum triglyceride (TG) level $(\mathrm{mg} / \mathrm{dL}) ;(9)$ incidence of hypoglycemia or hyperglycemia; and (10) serum total bilirubin (TB) level (mg/dL).

Two reviewers (K.K. and S.Y.K.) independently assessed the risk of bias for each study using the criteria outlined in the Cochrane Handbook for Systematic Reviews of Interventions [15] (Figure 2). We resolved disagreement by discussion.

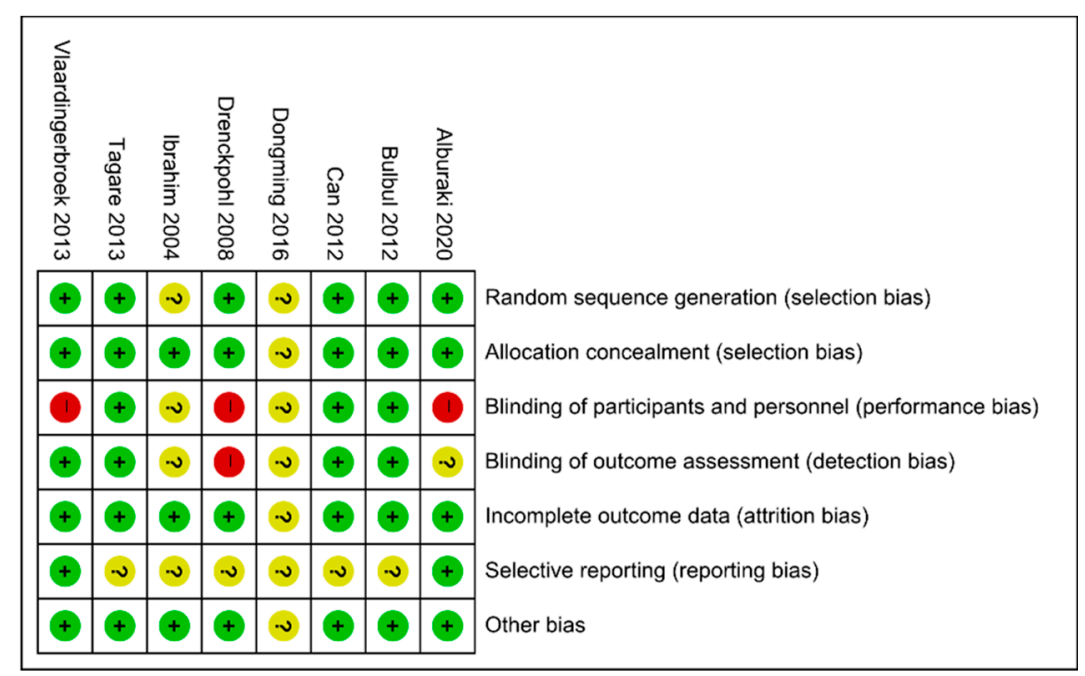

Figure 2. Risk of bias assessment of the randomized controlled trials included meta-analysis.

\subsection{Data Analysis}

Review Manager 5.4 (The Cochrane Collaboration, London, UK) was used to perform analyses. To analyze the treatment effect and calculate a pooled mean of outcomes reported in more than two studies, the Mantel-Haenszel method was used for categorical outcomes, and the inverse variance method was used for continuous outcomes. All tests were two tailed, and a $p$ value of less than 0.05 was deemed statistically significant. We used the $I^{2}$ statistic to assess heterogeneity in the results of individual studies $\left(I^{2}>50 \%\right.$ was used as a threshold indicating significant heterogeneity). We conducted sensitivity analyses when heterogeneity was distinguished. This was achieved by removing a study from the analysis to determine changes in $I^{2}$ values and assess which studies play a significant role resulting in heterogeneity [16].

\section{Results}

\subsection{Systematic Literature Search Results}

A total of 8972 citations were initially screened in the databases, and 6198 individual publications were identified. Among these, 6145 studies were excluded after reviewing 
titles and abstracts, leaving 53 articles for full text review for eligibility (Figure 1). Finally, nine studies were selected with proper eligibility [9,17-24]. One study [20], which was a planned follow-up of a previous RCT [19], was based on the same population and was therefore considered an identical study. Therefore, eight publications were included in our final meta-analysis [9,17-19,21-24].

\subsection{Sample Characteristics}

Overall, 520 infants were included in our meta-analysis: 262 infants in the early high IVLE groups and 258 infants in the control groups. IVLE was initiated between $2 \mathrm{~h}$ and $24 \mathrm{~h}$ after birth. The starting dose was equal to $1.5 \mathrm{~g} / \mathrm{kg} /$ day or more and was increased by $0.5-1.0 \mathrm{~g} / \mathrm{kg} /$ day over $1-2$ days to a maximum of $2.0-3.0 \mathrm{~g} / \mathrm{kg} /$ day in the intervention groups, which means that the administration dose of IVLE reached equal to or more than $1.5 \mathrm{~g} / \mathrm{kg}$ / day within $24 \mathrm{~h}$ after birth. On the other hand, IVLE was started between $12 \mathrm{~h}$ and 2 days after birth with a dose of $0.5-1.4 \mathrm{~g} / \mathrm{kg} /$ day in the control groups, and the maximum doses were lower than $1.5 \mathrm{~g} / \mathrm{kg} /$ day at $24 \mathrm{~h}$ after birth (Table 1 ). The risk of bias of the included studies was evaluated and is described in Figure 2. Follow-up was described in one RCT $[19,20]$. The characteristics of the included studies assessing the effect of early high IVLE ( $\geq 1.5 \mathrm{~g} / \mathrm{kg} /$ day) versus control and early low ( $<1.5 \mathrm{~g} / \mathrm{kg} /$ day) IVLE supplementation are presented in Table 2. 
Table 1. Characteristics of studies included in the meta-analysis.

\begin{tabular}{|c|c|c|c|c|c|c|c|}
\hline \multirow{2}{*}{ Study } & \multicolumn{5}{|c|}{ Characteristics } & \multicolumn{2}{|c|}{ Intervention (IV Lipid Intake) } \\
\hline & Country & Study Period & Design & Population & $n$ & Experimental Group & Control Group \\
\hline Alburaki, $2020[9]$ & Canada & $\begin{array}{l}\text { Aug } 2018 \text { to Oct } \\
2019\end{array}$ & $\mathrm{RCT}$ & $\begin{array}{c}\text { PT infants with } \mathrm{BW}<1500 \mathrm{~g} \\
\text { and }<32 \text { weeks GA }\end{array}$ & 83 & $\begin{array}{l}\text { Started } 2 \mathrm{~g} / \mathrm{kg} / \text { day within } 12 \mathrm{~h} \text { of } \\
\text { birth, increased to } 3 \mathrm{~g} / \mathrm{kg} / \text { day the } \\
\text { next day }\end{array}$ & $\begin{array}{l}\text { Started } 0.5 \mathrm{~g} / \mathrm{kg} / \text { day } \\
(\mathrm{BW}<1000 \mathrm{~g}) \text { or } 1 \mathrm{~g} / \mathrm{kg} / \text { day } \\
(\mathrm{BW} \geq 1000 \mathrm{~g} \text { ) between } 12-24 \mathrm{~h} \text {, } \\
\text { and advancing to } 3 \mathrm{~g} / \mathrm{kg} / \text { day } \\
\text { (increment } 0.5 \mathrm{~g} / \mathrm{kg} / \text { day) }\end{array}$ \\
\hline Dongming 2016 [17] & China & $\begin{array}{l}\text { June } 2013 \text { to June } \\
2015\end{array}$ & $\mathrm{RCT}$ & PT infants with BW $<1500 \mathrm{~g}$ & 80 & $\begin{array}{l}\text { Started } 1.5 \mathrm{~g} / \mathrm{kg} / \text { day within } 24 \mathrm{~h} \\
\text { of birth and advancing to } \\
3 \mathrm{~g} / \mathrm{kg} / \text { day (increment, } \\
0.5 \mathrm{~g} / \mathrm{kg} / \text { day) }\end{array}$ & $\begin{array}{l}\text { Only glucose within } 3 \text { days and } \\
\text { started same parenteral nutrition } \\
\text { after day } 3 \text {. }\end{array}$ \\
\hline Tagare, 2013 [18] & India & $\begin{array}{l}\text { Oct } 2009 \text { to Mar } \\
2010\end{array}$ & $\mathrm{RCT}$ & $\begin{array}{c}\text { PT infants with BW }<1500 \mathrm{~g} \\
\text { and }<32 \text { weeks GA }\end{array}$ & 34 & $\begin{array}{l}\text { Started with } 2 \mathrm{~g} / \mathrm{kg} / \text { day within } \\
24 \text { h of birth, remained same } \\
\text { thereafter }\end{array}$ & $\begin{array}{l}\text { Started } 1 \mathrm{~g} / \mathrm{kg} / \text { day at day } 3, \\
\text { remained same thereafter }\end{array}$ \\
\hline $\begin{array}{l}\text { Vlaardingerbroek, } \\
2013 \text { [19] }\end{array}$ & Netherland & Dec 2008 to Jan 2012 & $\mathrm{RCT}$ & PT infants with $\mathrm{BW}<1500 \mathrm{~g}$ & $97^{1}$ & $\begin{array}{l}\text { Started } 2 \mathrm{~g} / \mathrm{kg} / \text { day immediately } \\
\text { advancing to } 3 \mathrm{~g} / \mathrm{kg} / \text { day, day } 2 \text {. }\end{array}$ & $\begin{array}{l}\text { Started } 1.4 \mathrm{~g} / \mathrm{kg} / \text { day at day } 2, \\
\text { next day increased to } \\
2.8 \mathrm{~g} / \mathrm{kg} / \text { day }\end{array}$ \\
\hline Bulbul, 2012 [21] & Turkey & & $\mathrm{RCT}$ & $\begin{array}{l}\text { PT infants with } 750 \mathrm{~g}<\mathrm{BW} \\
<1500 \mathrm{~g} \text { and }<32 \text { weeks GA }\end{array}$ & 41 & $\begin{array}{l}\text { Started } 3 \mathrm{~g} / \mathrm{kg} / \text { day, on day } 1 \text {, } \\
\text { remained same thereafter }\end{array}$ & $\begin{array}{l}\text { Started } 1.0 \mathrm{~g} / \mathrm{kg} / \text { day at day } 3, \\
\text { advancing up to } 3 \mathrm{~g} / \mathrm{kg} / \text { day } \\
\text { (increment } 1.0 \mathrm{~g} / \mathrm{kg} / \text { day) }\end{array}$ \\
\hline Can, 2012 [22] & Turkey & $\begin{array}{c}\text { Feb } 2009 \text { to May } \\
2010\end{array}$ & $\mathrm{RCT}$ & $\begin{array}{c}\text { PT infants with }<34 \text { weeks } \\
\text { GA }\end{array}$ & 53 & $\begin{array}{l}\text { Started } 2 \mathrm{~g} / \mathrm{kg} / \text { day on day } 1, \\
\text { advancing to } 3.0 \mathrm{~g} / \mathrm{kg} / \text { day on } \\
\text { day } 2 .\end{array}$ & $\begin{array}{l}\text { Started } 1.0 \mathrm{~g} / \mathrm{kg} / \text { day on day } 1 \text {, } \\
\text { advancing up to } 3 \mathrm{~g} / \mathrm{kg} / \text { day } \\
\text { (increment } 1.0 \mathrm{~g} / \mathrm{kg} / \text { day) }\end{array}$ \\
\hline Drenckpohl, 2008 [23] & Illinois, US & $\begin{array}{l}\text { June } 2005 \text { to Sep } \\
2009\end{array}$ & $\mathrm{RCT}$ & $\begin{array}{c}\text { PT infants with } 750 \mathrm{~g}<\mathrm{BW} \\
<1500 \mathrm{~g}\end{array}$ & 100 & $\begin{array}{l}\text { Started } 2 \mathrm{~g} / \mathrm{kg} / \text { day on day } 1 \text {, } \\
\text { advancing to } 3 \mathrm{~g} / \mathrm{kg} / \text { day } \\
\text { (increment } 0.5 \mathrm{~g} / \mathrm{kg} / \text { day) }\end{array}$ & $\begin{array}{l}\text { Started } 0.5 \mathrm{~g} / \mathrm{kg} / \text { day on day } 1 \text {, } \\
\text { advancing up to } 3 \mathrm{~g} / \mathrm{kg} / \text { day } \\
\text { (increment } 0.5 \mathrm{~g} / \mathrm{kg} / \text { day) }\end{array}$ \\
\hline Ibranhim, 2004 [24] & Louisiana, US & $\begin{array}{l}\text { July } 2001 \text { to Apr } \\
2002\end{array}$ & $\mathrm{RCT}$ & $\begin{array}{c}\text { PT infants with } 500 \mathrm{~g}<\mathrm{BW} \\
<1250 \mathrm{~g} \text { and } 24 \leq \mathrm{GA}<32 \\
\text { weeks }\end{array}$ & 32 & $\begin{array}{l}\text { Started } 3 \mathrm{~g} / \mathrm{kg} / \text { day within } 2 \mathrm{~h} \\
\text { after birth, remained same } \\
\text { thereafter }\end{array}$ & $\begin{array}{l}\text { started } 0.5 \mathrm{~g} / \mathrm{kg} / \text { day at } 48 \mathrm{~h} \text { after } \\
\text { birth, advancing up to } 3 \mathrm{~g} / \mathrm{kg} / \text { day } \\
\text { (increment } 0.5 \mathrm{~g} / \mathrm{kg} / \text { day) }\end{array}$ \\
\hline
\end{tabular}

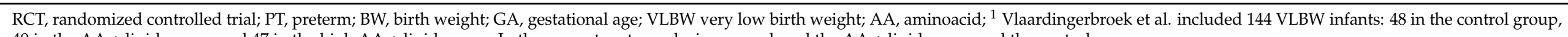

49 in the AA + lipid group, and 47 in the high AA + lipid group. In the current meta-analysis, we analyzed the AA + lipid group and the control group. 
Table 2. Baseline characteristics and outcome measures of infants in studies analyzed.

\begin{tabular}{|c|c|c|c|c|c|c|c|c|c|c|c|c|c|c|c|c|c|c|c|c|c|}
\hline $\begin{array}{l}\text { First Author, } \\
\text { Year }\end{array}$ & $n$ & Male & GA & BW & $\begin{array}{c}\mathrm{Wt} \\
\text { Gain } \\
\text { Rate }\end{array}$ & $\begin{array}{r}\text { Max } \\
\text { \%Age } \\
\text { of wt } \\
\text { Loss } \\
\end{array}$ & $\begin{array}{c}\text { Time } \\
\text { to } \\
\text { Regain } \\
\text { BW }\end{array}$ & $\begin{array}{l}\text { Wt } \\
\text { Near } \\
\text { TEA }\end{array}$ & $\begin{array}{c}\text { HC } \\
\text { Near } \\
\text { TEA }\end{array}$ & EUGR & Death & $\begin{array}{l}\text { NICU } \\
\text { Stay }\end{array}$ & BPD/CLD & $\begin{array}{l}\text { NEC } \\
\geq 2\end{array}$ & $\begin{array}{l}\text { Proven } \\
\text { Sepsis }\end{array}$ & $\begin{array}{l}\text { IVH } \\
\geq 3\end{array}$ & ROP & $\begin{array}{c}\text { Hy- } \\
\text { perTG }\end{array}$ & $\begin{array}{c}\text { Hypo- } \\
\text { glycemia }\end{array}$ & $\begin{array}{c}\text { Hyper- } \\
\text { glycemia }\end{array}$ & $\begin{array}{c}\text { Serum } \\
\text { TB }\end{array}$ \\
\hline & & $\begin{array}{c}n \\
(\%)\end{array}$ & wk & $\mathrm{g}$ & $\mathrm{g} / \mathrm{kg} /$ day & $\%$ & Day & $\mathrm{g}$ & $\mathrm{cm}$ & $\begin{array}{c}\mathrm{n} \\
(\%)\end{array}$ & $\mathrm{n}$ & day & $\mathrm{n}(\%)$ & $\mathrm{n}(\%)$ & $\mathrm{n}(\%)$ & $\mathrm{n}(\%)$ & $\mathrm{n}(\%)$ & $\mathrm{n}(\%)$ & $\mathrm{n}(\%)$ & $\mathrm{n}(\%)$ & $\mathrm{mg} / \mathrm{dL}$ \\
\hline \multicolumn{22}{|c|}{ Intervention } \\
\hline $\begin{array}{c}\text { Alburaki, } 2020 \\
{[9]}\end{array}$ & 45 & $\begin{array}{c}28 \\
(62)\end{array}$ & $\begin{array}{c}27.1 \pm \\
2.3\end{array}$ & $\begin{array}{c}1019 \pm \\
271\end{array}$ & $\begin{array}{c}15.2 \pm \\
2.0^{1}\end{array}$ & $\begin{array}{l}10.4 \pm \\
3.6^{2}\end{array}$ & $\begin{array}{c}10.5 \\
(8,13)\end{array}$ & $\begin{array}{r}2278 \pm \\
303^{3}\end{array}$ & $\begin{array}{c}31.3^{ \pm} \\
1.5^{3}\end{array}$ & $\begin{array}{c}17 \\
(38.6) \\
3\end{array}$ & 1 & $\begin{array}{c}82 \\
(49.5,107)\end{array}$ & $\begin{array}{c}15 \\
(33.3)^{4}\end{array}$ & $1(2.2)$ & $3(6.7)$ & $4(8.9)$ & $6(25)^{5}$ & $\begin{array}{c}15 \\
(33.3)^{6}\end{array}$ & $\begin{array}{c}14 \\
(31.1)^{7}\end{array}$ & $0^{8}$ & $\begin{array}{l}8.42 \pm \\
1.52{ }^{9}\end{array}$ \\
\hline $\begin{array}{c}\text { Dongming, } 2016 \\
{[17]}\end{array}$ & 40 & $\begin{array}{l}24 \\
(60) \\
\end{array}$ & $\begin{array}{c}30.2 \\
(28,34) \\
\end{array}$ & $\begin{array}{c}1140 \pm \\
220\end{array}$ & - & $\begin{array}{c}7.7 \pm \\
1.5 \\
\end{array}$ & $\begin{array}{c}8.2 \pm \\
2.4 \\
\end{array}$ & - & - & - & - & - & - & - & - & - & - & - & - & 1 & - \\
\hline Tagare, 2013 [18] & 17 & & $\begin{array}{c}30.5 \pm \\
2.6\end{array}$ & $\begin{array}{c}1162 \pm \\
224\end{array}$ & - & - & $\begin{array}{c}9.5 \pm \\
6.7\end{array}$ & - & - & - & 2 & $\begin{array}{c}19.5 \pm \\
13.3\end{array}$ & - & - & - & - & - & - & - & - & $\begin{array}{l}6.7 \pm \\
3.1^{10}\end{array}$ \\
\hline $\begin{array}{c}\text { Vlaardingerbroek, } \\
2013 \text { [19] }\end{array}$ & 49 & $\begin{array}{c}19 \\
(39) \\
\end{array}$ & $\begin{array}{c}27.2 \pm \\
2.2 \\
\end{array}$ & $\begin{array}{c}876 \pm \\
209 \\
\end{array}$ & $\begin{array}{r}25.0 \pm \\
5.2^{11} \\
\end{array}$ & - & $\begin{array}{c}8(5,12), \\
\text { ns }\end{array}$ & - & - & - & 10 & $\begin{array}{c}86.5 \pm \\
29.1 \\
\end{array}$ & $11_{12}^{(22)}$ & $4(8)$ & 17 (35) & $2(4)$ & $0(0)$ & $27 \%^{13}$ & - & $24 \%^{8}$ & $\begin{array}{c}8.26 \pm \\
2.2^{9} \\
\end{array}$ \\
\hline Bulbul, 2012 [21] & 22 & $70 \%$ & $\begin{array}{c}29.1 \pm \\
1.1 \\
\end{array}$ & $\begin{array}{c}1316 \pm \\
247\end{array}$ & - & - & $\begin{array}{c}12.5 \pm \\
5.4\end{array}$ & $\begin{array}{c}2210 \pm \\
91^{14}\end{array}$ & $\begin{array}{l}32.1 \pm \\
2.3^{14} \\
\end{array}$ & - & - & $\begin{array}{c}34.4 \pm \\
18.1 \\
\end{array}$ & - & $2(9)$ & $1(4.5)$ & 0 & - & - & - & - & $\begin{array}{l}7.2 \pm \\
0.6^{10} \\
\end{array}$ \\
\hline Can, 2012 [22] & 25 & $\begin{array}{l}16 \\
(64)\end{array}$ & $\begin{array}{c}31.3 \\
(27,33)\end{array}$ & $\begin{array}{c}1622 \pm \\
276\end{array}$ & - & - & $\begin{array}{c}12.7 \pm \\
2.8\end{array}$ & $\begin{array}{r}3180 \pm \\
474^{15}\end{array}$ & $\begin{array}{l}34.7 \pm \\
1.5^{15}\end{array}$ & $\begin{array}{l}13 \\
(52) \\
15\end{array}$ & 1 & $\begin{array}{l}28 \pm \\
15.6\end{array}$ & $1(4)^{16}$ & $1(4)$ & - & - & $2(8)^{17}$ & - & - & - & - \\
\hline$\underset{[24]}{\text { Ibranhim, }} 2004$ & 16 & $\begin{array}{l}10 \\
(63)\end{array}$ & $\begin{array}{c}27 \pm \\
1.6\end{array}$ & $\begin{array}{c}846 \pm \\
261\end{array}$ & - & - & - & - & - & - & 1 & - & $\begin{array}{c}9 \\
(56.25) \\
16\end{array}$ & - & 6 & 4 & 2 & - & - & - & $\begin{array}{l}7.7 \pm \\
0.42^{21}\end{array}$ \\
\hline \multicolumn{22}{|c|}{ Control } \\
\hline $\begin{array}{c}\text { Alburaki, } 2020 \\
{[9]}\end{array}$ & 38 & $\begin{array}{c}25 \\
(66)\end{array}$ & $\begin{array}{c}27.3 \pm \\
2.4\end{array}$ & $\begin{array}{l}1011 \pm \\
250\end{array}$ & $\begin{array}{c}15.3^{ \pm} \\
3.5^{1}\end{array}$ & $\begin{array}{l}12.7 \pm \\
4.6^{2}\end{array}$ & $\begin{array}{c}11.5 \\
(8,16)\end{array}$ & $\begin{array}{r}2165 \pm \\
301^{3}\end{array}$ & $\begin{array}{c}30.5 \pm \\
1.4^{3}\end{array}$ & $\begin{array}{c}25 \\
(67.6) \\
3\end{array}$ & 1 & $\begin{array}{c}80.5 \\
(58,96)\end{array}$ & $8 \underset{4}{8(21.1)}$ & $1(2.6)$ & $2(5.3)$ & $2(5.3)$ & $6 \underset{5}{6(26.1)}$ & $7 \underset{6}{(18.4)}$ & $\begin{array}{c}11 \\
(28.9)^{7}\end{array}$ & $0^{8}$ & $\begin{array}{c}8.65 \pm \\
1.7^{9}\end{array}$ \\
\hline $\begin{array}{c}\text { Dongming, } \\
{[17]}\end{array}$ & 40 & $\begin{array}{c}25 \\
(63) \\
\end{array}$ & $\begin{array}{c}30.4 \\
(28,34)\end{array}$ & $\begin{array}{c}1148 \pm \\
216\end{array}$ & - & $\begin{array}{c}10.6 \pm \\
3.3\end{array}$ & $\begin{array}{c}11.6 \pm \\
3.0\end{array}$ & - & - & - & - & - & - & - & - & - & - & - & - & 3 & - \\
\hline Tagare, 2013 [18] & 17 & & $\begin{array}{c}32.1 \pm \\
2.8 \\
\end{array}$ & $\begin{array}{c}1264 \pm \\
194 \\
\end{array}$ & - & - & $\begin{array}{c}11.5 \pm \\
6.7 \\
\end{array}$ & - & - & - & 1 & $\begin{array}{c}20.2 \pm \\
12.9 \\
\end{array}$ & - & - & - & - & - & - & - & - & $\begin{array}{l}7.2 \pm \\
2.5^{10}\end{array}$ \\
\hline $\begin{array}{l}\text { Vlaardingerbroek, } \\
2013 \text { [19] }\end{array}$ & 48 & $\begin{array}{c}25 \\
(52)\end{array}$ & $\begin{array}{c}27.8 \pm \\
2.3\end{array}$ & $\begin{array}{c}843 \pm \\
224\end{array}$ & $\begin{array}{l}25.8 \pm \\
8.1^{11}\end{array}$ & - & $\begin{array}{c}8(5,12), \\
\text { ns }\end{array}$ & - & - & - & 5 & $\begin{array}{c}91.0 \pm \\
39.9\end{array}$ & $\begin{array}{c}16(33) \\
12\end{array}$ & $2(4)$ & $8(17)$ & $1(2)$ & $2(4)$ & $44 \%^{13}$ & - & $6 \%^{8}$ & $\begin{array}{l}8.77 \pm \\
1.88^{9}\end{array}$ \\
\hline
\end{tabular}


Table 2. Cont.

\begin{tabular}{|c|c|c|c|c|c|c|c|c|c|c|c|c|c|c|c|c|c|c|c|c|c|}
\hline $\begin{array}{l}\text { First Author, } \\
\text { Year }\end{array}$ & $n$ & Male & GA & BW & $\begin{array}{c}\mathrm{Wt} \\
\text { Gain } \\
\text { Rate }\end{array}$ & $\begin{array}{c}\text { Max } \\
\text { \%Age } \\
\text { of wt } \\
\text { Loss }\end{array}$ & $\begin{array}{c}\text { Time } \\
\text { to } \\
\text { Regain } \\
\text { BW }\end{array}$ & $\begin{array}{l}\mathrm{Wt} \\
\text { Near } \\
\text { TEA }\end{array}$ & $\begin{array}{l}\text { HC } \\
\text { Near } \\
\text { TEA }\end{array}$ & EUGR & Death & $\begin{array}{l}\text { NICU } \\
\text { Stay }\end{array}$ & BPD/CLD & $\begin{array}{l}\text { NEC } \\
\geq 2\end{array}$ & $\begin{array}{l}\text { Proven } \\
\text { Sepsis }\end{array}$ & $\begin{array}{l}\text { IVH } \\
\geq 3\end{array}$ & ROP & $\begin{array}{c}\text { Hy- } \\
\text { perTG }\end{array}$ & $\begin{array}{c}\text { Hypo- } \\
\text { glycemia }\end{array}$ & $\begin{array}{l}\text { Hyper- } \\
\text { glycemia }\end{array}$ & $\begin{array}{c}\text { Serum } \\
\text { TB }\end{array}$ \\
\hline Bulbul, 2012 [21] & 22 & $52 \%$ & $\begin{array}{c}29.4 \pm \\
1.8\end{array}$ & $\begin{array}{c}1355 \pm \\
237\end{array}$ & - & - & $\begin{array}{c}10.2 \pm \\
3.9\end{array}$ & $\begin{array}{l}2155 \pm \\
180^{14}\end{array}$ & $\begin{array}{l}31.2 \pm \\
2.1^{14}\end{array}$ & - & - & $\begin{array}{c}33.5 \pm \\
19.4\end{array}$ & - & $1(4.5)$ & $1(4.5)$ & 0 & - & - & - & - & $\begin{array}{l}7.7 \pm \\
0.5^{10}\end{array}$ \\
\hline Can, 2012 [22] & 25 & $\begin{array}{l}15 \\
(60)\end{array}$ & $\begin{array}{c}31.4 \\
(27,33)\end{array}$ & $\begin{array}{c}1598 \pm \\
346\end{array}$ & - & - & $\begin{array}{c}14.2 \pm \\
3.0\end{array}$ & $\begin{array}{l}2992 \pm \\
445^{15}\end{array}$ & $\begin{array}{l}33.6 \pm \\
1.5^{15}\end{array}$ & $\begin{array}{c}22 \\
(88) \\
15\end{array}$ & 2 & $\begin{array}{c}29.9 \pm \\
4.8\end{array}$ & $1(4)$ & $2(8)$ & - & - & $\underset{17}{8(32)}$ & - & - & - & - \\
\hline $\begin{array}{l}\text { Drenckpohl, } \\
2008 \text { [23] }\end{array}$ & 52 & $55.8 \%$ & $\begin{array}{c}28.6 \pm \\
1.8\end{array}$ & $\begin{array}{c}1134 \pm \\
223\end{array}$ & - & - & $\begin{array}{c}12.9 \pm \\
3.8\end{array}$ & $\begin{array}{l}1946 \pm \\
771^{14}\end{array}$ & $31 \pm 2$ & $\begin{array}{c}43 \\
(83) \\
14\end{array}$ & 3 & $\begin{array}{l}50.58 \\
\pm 33\end{array}$ & $7 \underset{16}{(14)}$ & $7(14)$ & - & $\cos _{18}(21)$ & $\underset{19}{12(23)}$ & $2(4)^{20}$ & - & $10 \%^{8}$ & - \\
\hline $\begin{array}{l}\text { Ibranhim, } \\
{[24]}\end{array}$ & 16 & $\begin{array}{c}9 \\
(56)\end{array}$ & $\begin{array}{c}26.8 \pm \\
1.5\end{array}$ & $\begin{array}{c}968 \pm \\
244\end{array}$ & - & - & - & - & - & - & 2 & - & $\begin{array}{c}8(50) \\
16\end{array}$ & - & 7 & 5 & 3 & - & - & - & $\begin{array}{l}6.2 \pm \\
0.43^{21}\end{array}$ \\
\hline
\end{tabular}

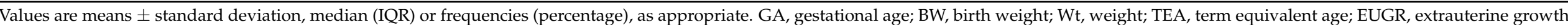

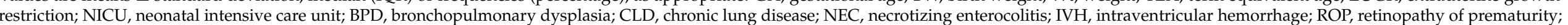

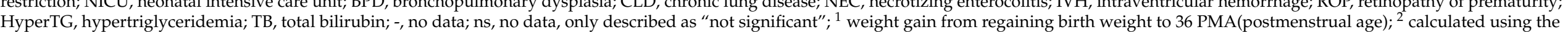

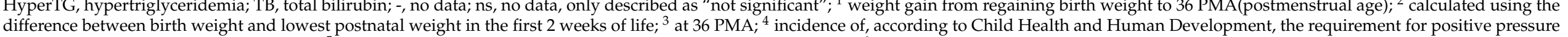

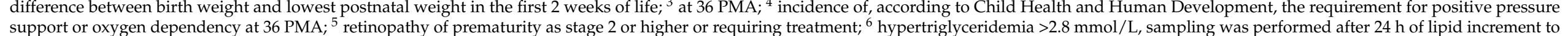

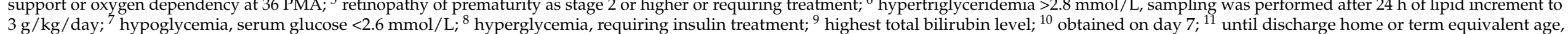

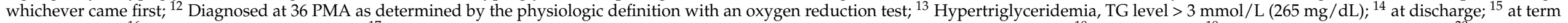

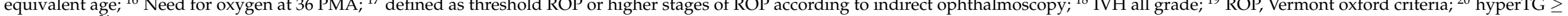
$201 \mathrm{mg} / \mathrm{dL} ;{ }^{21}$ Mean, during the first 7 days. 


\subsection{Primary Outcome Measures}

The weight gain rate was measured in two studies [9,19], We conducted a metaanalysis of these two studies, which showed no differences between the early high IVLE group and control groups (Figure 3a). However, there was a lack of consistency in the timing to measure weight. Alburaki et al. [9] reported mean weight gain from regaining birth weight to 36 weeks PMA, meanwhile the study of Vlaardingerbroek et al. [19], where growth until discharge home or until 40 weeks PMA was measured. Alburaki et al. [9] and Dongming et al. [17] documented the proportion of postnatal weight loss. A metaanalysis that included these two studies showed a significant favorable association of early high IVLE administration (mean difference [MD]: -2.73 ; 95\% confidence interval [CI]: $-3.69,-1.78 ; p<0.00001 ; n=163$ ) (Figure 3b), but in two RCTs, the timeline of this outcome was inconsistent. Two studies were excluded, and the time to regain birth weight was reported in the remaining six RCTs $[9,17,18,21-23]$. A meta-analysis including these studies discerned no significant differences between the intervention group and control group (Figure 3c). As a consequence of considerably high heterogeneity with an $I^{2}$ of $74 \%$, sensitivity analyses with the removal of several far-reaching studies were conducted; however, the heterogeneity was not improved. Growth parameters until discharge home or TEA were measured in four studies. Alburaki et al. [9] reported growth anthropometrics measured at 36 weeks PMA; on the other hand, Can et al. [22] measured at 40 weeks PMA, and Bulbul et al. [21] and Drenckpohl et al. [23] checked the weight of the infant at discharge to the home. In aggregate, a meta-analysis of mean body weight near TEA that included four previous studies was performed [9,21-23]. The result showed a significant difference that favored the intervention group, which means that early high IVLE administration has a better effect on growth than the control parameter (MD: 70.27; 95\% CI: 4.73, 135.81; $p=0.04$; $n=277$, Figure 3d). In the same four studies [9,21-23], the mean head circumference near TEA was measured and reported. A meta-analysis showed a significant difference in favor of the intervention group (MD: 0.67; 95\% CI: 0.25, 1.09; $p=0.002 ; n=277$; Figure 3e). Three studies reported the incidence of EUGR [9,22,23]; Alburaki et al. evaluated the infants at 36 weeks of PMA, Can et al. at 40 weeks of PMA, and Drenckpohl et al. at discharge. A meta-analysis of these three studies was possible and showed an association between early high parenteral lipid administration and a lower incidence of EUGR (relative risk [RR]: 0.27 ; 95\% CI: 0.15, $0.48 ; p<0.00001 ; n=233$, Figure 3f). 
(a) Weight gain rate, $\mathrm{g} / \mathrm{kg} / \mathrm{d}$

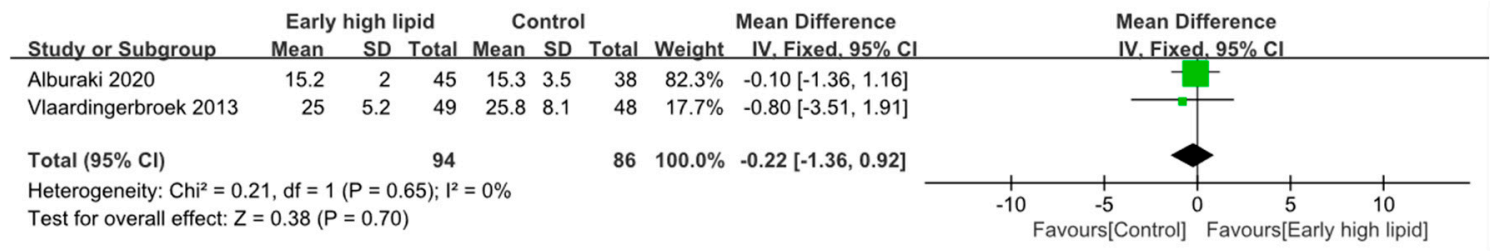

(b) Proportion of postnatal weight loss, \%

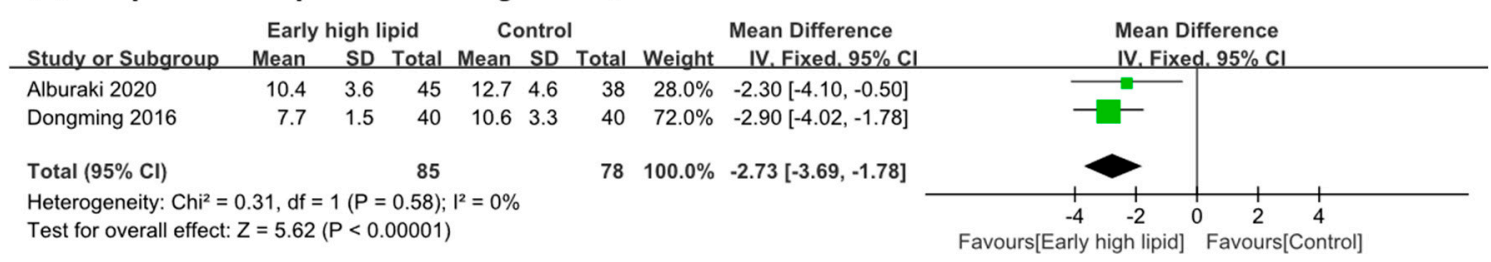

(c) Time to regain birth weight, days

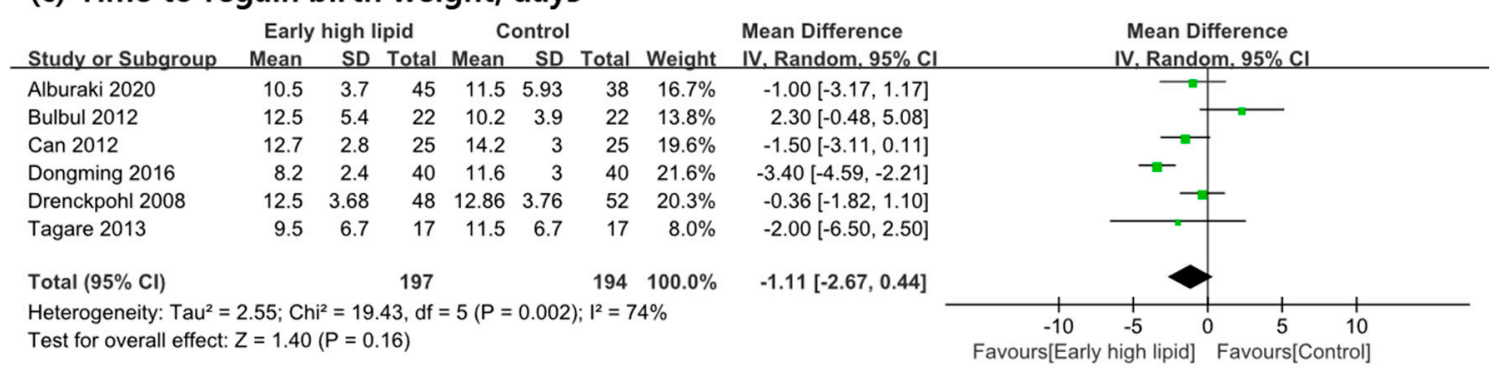

(d) Body weight near TEA, g

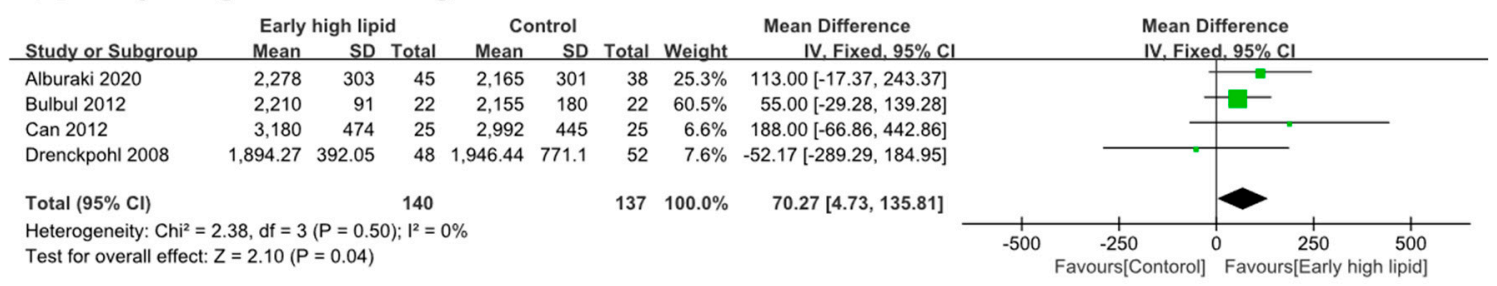

(e) Head circumference near TEA, cm

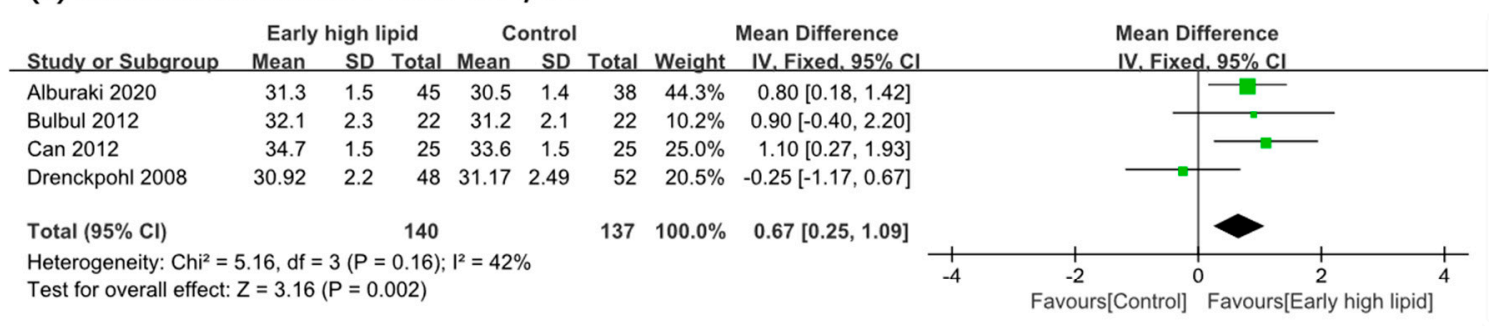

\section{(f) EUGR}

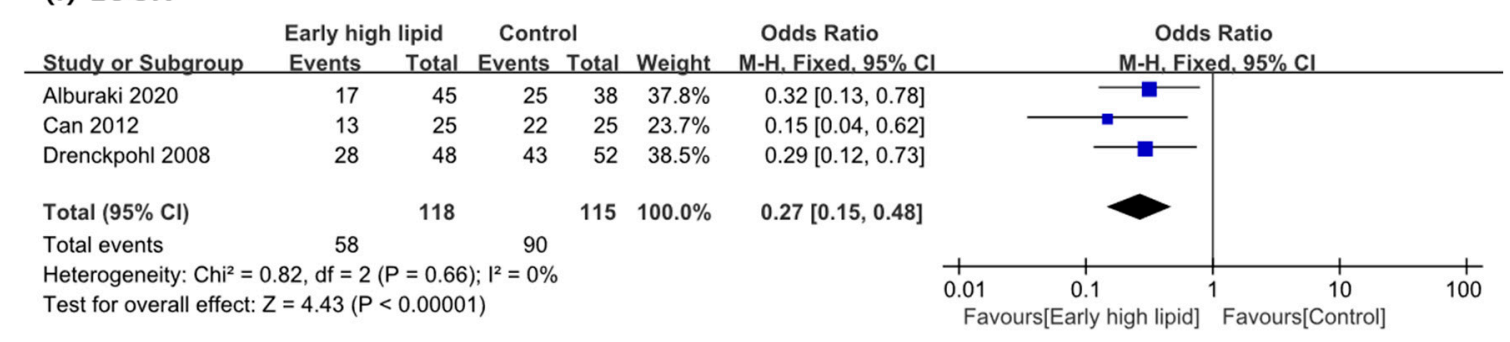

Figure 3. (a-f): Meta-analysis of the effects of early high IVLE that reached a lipid dose equal to or greater than $1.5 \mathrm{~g} / \mathrm{kg} / \mathrm{day}$ within the first $24 \mathrm{~h}$ on the growth of preterm infants compared with controls (random effects). IV, inverse variance; $\mathrm{M}-\mathrm{H}$, Mantel-Haenszel; CI, confidence interval; TEA, term equivalent age; EUGR, extrauterine growth restriction. 


\subsection{Secondary Outcome Measures}

In a subtotal of six studies $[9,18,19,22-24]$, the overall mortality was $7.5 \%(15 / 201)$ in the early high IVLE group and 7.1\% (14/198) in the control group. None of these studies reported a significant discrepancy in mortality between groups, and meta-analysis supported this finding. The length of NICU stay was reported in six RCTs $[9,18,19,21-23]$, and no significant effect of early high lipid to admission length was disclosed.

The incidence of BPD was reported in five studies. Alburaki et al. [9] diagnosed BPD on the basis of the National Institute of Child Health and Human Development workshop [25]. In the study of Vlaardingerbroek et al., this was diagnosed by physiologic definition with an oxygen reduction test [26]. In three studies by Can et al., Drenckpohl et al., and Ibrahim et al. [22-24], the incidence of requiring oxygen at a postmenstrual age of 36 weeks was described, and this could be used for the diagnosis of BPD or CLD. However, no significant effect of early high IVLE administration was confirmed in individual studies or in the current meta-analysis of these five studies ( $p=0.87$, Figure $4 \mathrm{c}$ ). The incidence of NEC was described in five studies [9,19,21-23]. At the individual study level, only one study reported a significantly higher NEC incidence in the control group than in the experimental group [23]. The other four individual studies showed no significant difference, which was underscored by the meta-analysis of five studies ( $p=0.28$, Figure $4 \mathrm{~d}$ ). Alburaki et al. [9] reported early onset sepsis; Vlaardingerbroek et al. [19] reported late onset sepsis with culture proven; and Bulbul et al. [21] and Ibrahim et al. [24] defined sepsis as a positive blood culture not specifying the timing. None of the original studies reported a significant difference in the incidence of sepsis, and this was confirmed by meta-analysis of four studies ( $p=0.13$, Figure 4e). The incidence of severe IVH was reported in the studies of Alburaki et al., Vlaardingerbroek et al., and Ibrahim et al. [9,19,24]. Again, no significant effect of early high IVLE administration was confirmed in individual studies or in this meta-analysis ( $p=0.64$, Figure $4 \mathrm{f}$ ). The incidence of ROP was described in five studies $[9,19,22-24]$, although the applied definitions in each study were diverse. A meta-analysis of five studies showed an effect of early high IVLE administration on the occurrence of ROP, which favored intervention (RR: $0.37 ; 95 \%$ CI: $0.18,0.73 ; p=0.004$; $n=359$, Figure $4 \mathrm{~g}$ ).

Blood samples of infants included in each RCT were obtained in all studies, and the current meta-analysis was evaluated three items: serum TG, TB, and glucose. Episodes of hypertriglyceridemia were counted in only two studies: Alburaki et al. [9] defined hypertriglyceridemia as serum TG level $>2.8 \mathrm{mmol} / \mathrm{L}(247.8 \mathrm{mg} / \mathrm{dL})$, and Drenckpohl et al. [23] as more than $200 \mathrm{mg} / \mathrm{dL}$ of serum TG. A meta-analysis of these two studies revealed a significant effect of early high lipid introduction on episodes of hypertriglyceridemia; hypertriglyceridemia was observed more often in the intervention group (RR: $2.72 ; 95 \%$ CI: 1.15, 6.43; $p=0.02 ; n=183$; Figure 4h). Episodes of hypoglycemia were documented in only one study by Alburaki et al. [9]; they defined hypoglycemia as blood sugar level $<2.6 \mathrm{mmol} / \mathrm{L}(46.8 \mathrm{mg} / \mathrm{dL})$ and were not significantly different between groups. In contrast, the incidence of hyperglycemia was noted in two studies [9,17]; however, Alburaki et al. reported that no patient required insulin treatment. Therefore, it was impossible to perform a meta-analysis because only one study remained. No significant effect on the serum bilirubin level of early high IVLE administration was found in the meta-analysis of five studies $[9,18,19,21,24]\left(p=0.84\right.$ and $\left.I^{2}=96 \%\right)$. There was considerably high heterogeneity with a lack of consistency in the timing of blood sampling in each study: highest level $[9,19]$, at the 7 th day of life $[18,21]$, and mean value during the first week [24]. To improve the $I^{2}$ statics, a subgroup meta-analysis including two studies about the effect on the highest serum total bilirubin $[9,19]$ was conducted and showed no significant effect of early high lipid administration ( $p=0.20, I^{2}=0$, supplementary Figure S1). 
(a) Death before NICU discharge

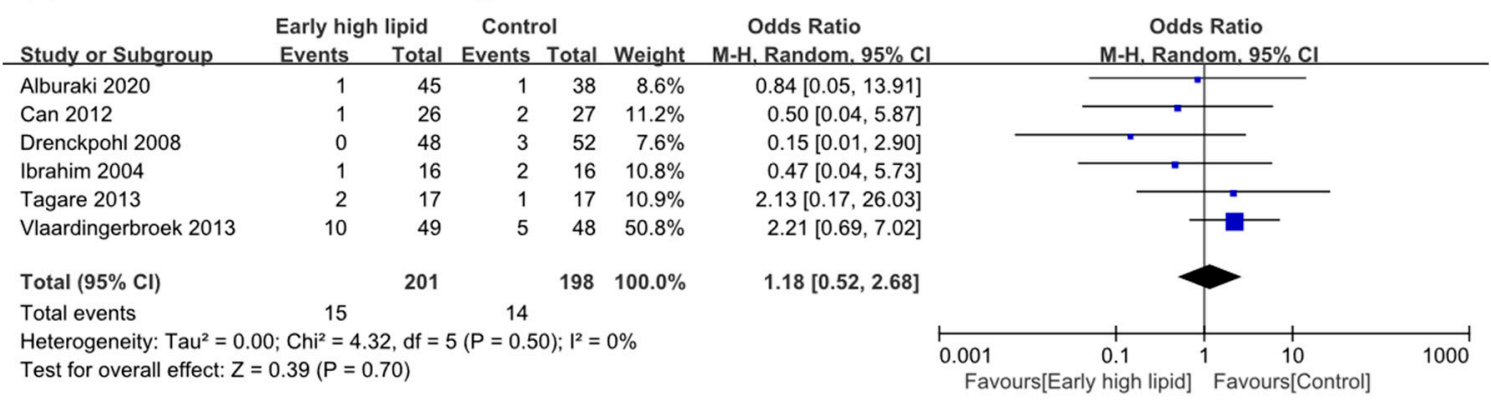

\section{(b) Length of NICU stay, days}

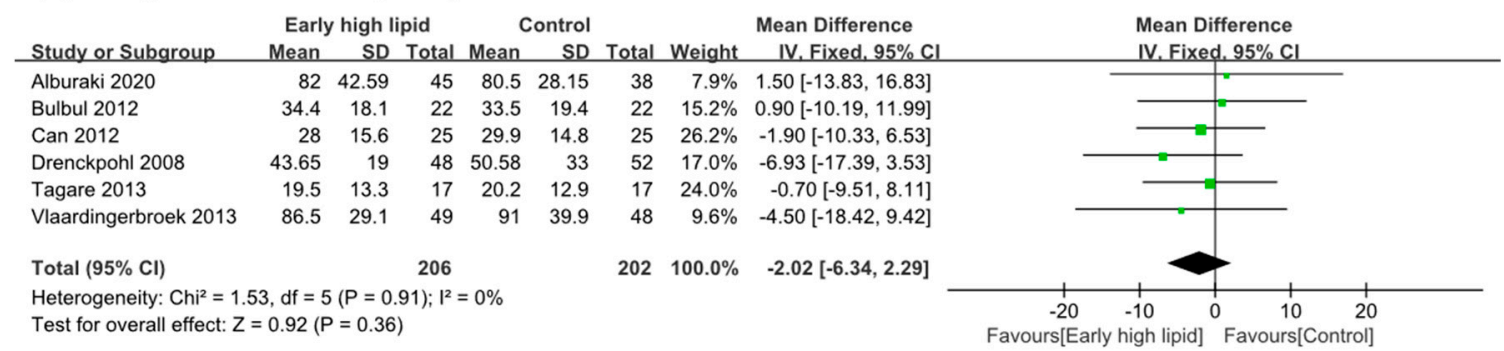

\section{(c) BPD}

\begin{tabular}{|c|c|c|c|c|c|}
\hline \multirow[b]{2}{*}{ Study or Subgroup } & \multicolumn{2}{|c|}{ Early high lipid } & \multicolumn{2}{|c|}{ Control } & \multirow[b]{2}{*}{ Weight } \\
\hline & Events & Total & Events & Total & \\
\hline Alburaki 2020 & 15 & 45 & 8 & 38 & $29.5 \%$ \\
\hline Can 2012 & 1 & 25 & 1 & 25 & $3.7 \%$ \\
\hline Drenckpohl 2008 & 4 & 48 & 7 & 52 & $17.4 \%$ \\
\hline Ibrahim 2004 & 9 & 14 & 8 & 15 & $13.2 \%$ \\
\hline Vlaardingerbroek 2013 & 11 & 49 & 16 & 48 & $36.2 \%$ \\
\hline Total $(95 \% \mathrm{Cl})$ & & 181 & & 178 & $100.0 \%$ \\
\hline Total events & 40 & & 40 & & \\
\hline Heterogeneity: $\operatorname{Tau}^{2}=0$ & $\mathrm{Chi}^{2}=3$ & $d f=$ & $=0.42$ & $1^{2}$ & \\
\hline
\end{tabular}

Odds Ratio

H. Random. 95\% C $1.88[0.69,5.08]$ $1.00[0.06,16.93]$ $0.58[0.16,2.14]$ $1.57[0.35,7.00]$ $0.58[0.24,1.42]$ $0.96[0.56,1.64]$ Test for overall effect: $Z=0.17(P=0.87)$

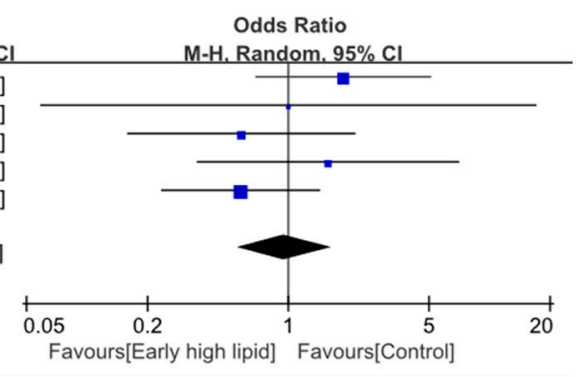

\section{(d) NEC $\geq$ stage 2}

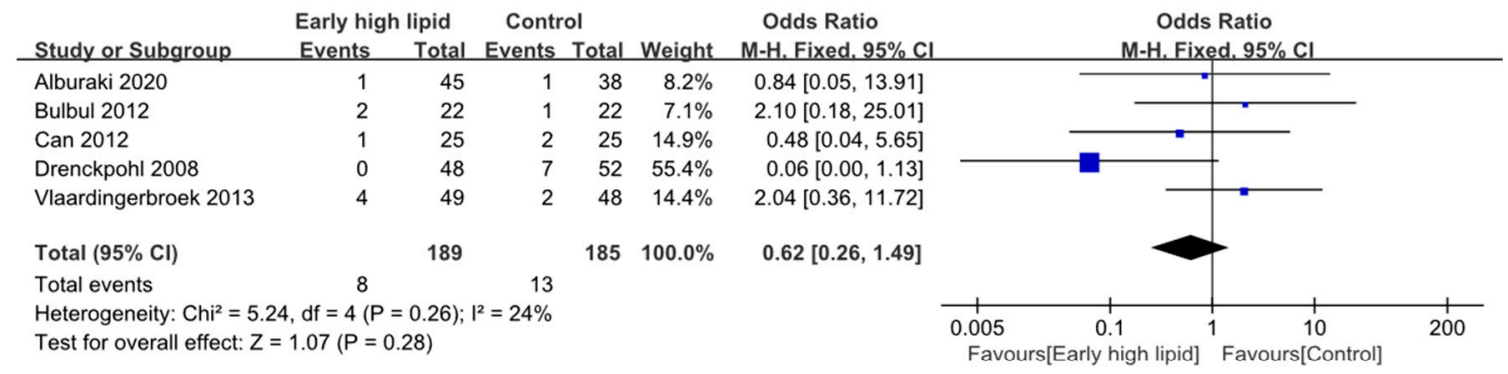

\section{(e) Culture proven sepsis}

\begin{tabular}{|c|c|c|c|c|c|c|c|c|c|c|}
\hline Study or Subgroup & $\begin{array}{l}\text { Early hig } \\
\text { Events }\end{array}$ & $\begin{array}{l}\text { lipid } \\
\text { Total }\end{array}$ & $\begin{array}{l}\text { Contro } \\
\text { Events }\end{array}$ & $\begin{array}{l}\text { ol } \\
\text { Total }\end{array}$ & Weight & $\begin{array}{l}\text { Odds Ratio } \\
\text { M-H. Fixed. } 95 \% \mathrm{CI}\end{array}$ & & $\begin{array}{l}\text { Odds Ratio } \\
\text { M-H. Fixed. } 95 \% \mathrm{Cl}\end{array}$ & & \\
\hline Alburaki 2020 & 3 & 45 & 2 & 38 & $16.7 \%$ & $1.29[0.20,8.13]$ & & 等 & & \\
\hline Bulbul 2012 & 1 & 22 & 1 & 22 & $7.9 \%$ & $1.00[0.06,17.07]$ & & & & \\
\hline Ibrahim 2004 & 6 & 14 & 7 & 15 & $31.9 \%$ & $0.86[0.20,3.71]$ & & & & \\
\hline Vlaardingerbroek 2013 & 17 & 49 & 8 & 48 & $43.6 \%$ & $2.66[1.02,6.94]$ & & & & \\
\hline Total $(95 \% \mathrm{Cl})$ & & 130 & & 123 & $100.0 \%$ & $1.72[0.86,3.47]$ & & & & \\
\hline Total events & 27 & & 18 & & & & & & & \\
\hline \multirow{2}{*}{\multicolumn{7}{|c|}{$\begin{array}{l}\text { Heterogeneity: } \mathrm{Chi}^{2}=1.89, \mathrm{df}=3(\mathrm{P}=0.60) ; \mathrm{I}^{2}=0 \% \\
\text { Test for overall effect: } Z=1.52(P=0.13)\end{array}$}} & 0.01 & 1 & 10 & 100 \\
\hline & & & & & & & Favours[Early & high lipid] & ontrol] & \\
\hline
\end{tabular}

Figure 4. Cont. 


\section{(f) Severe IVH}

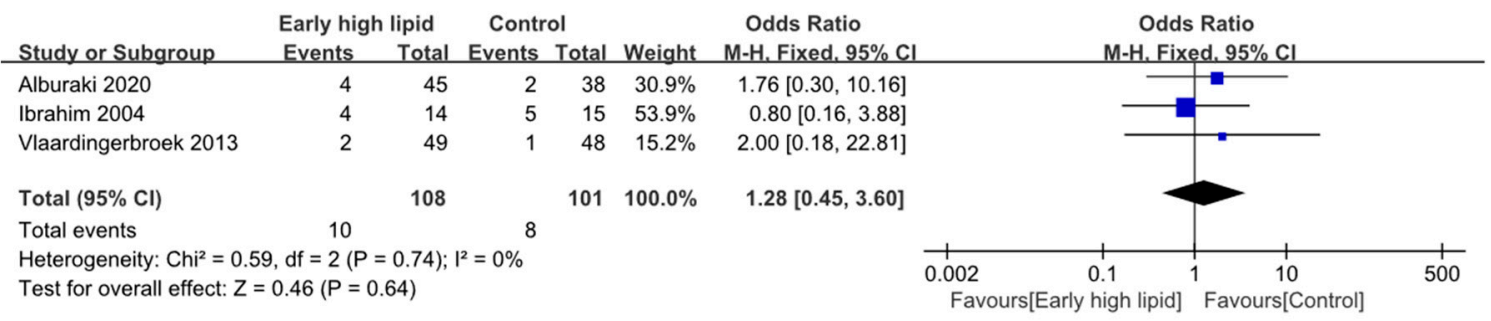

(g) ROP

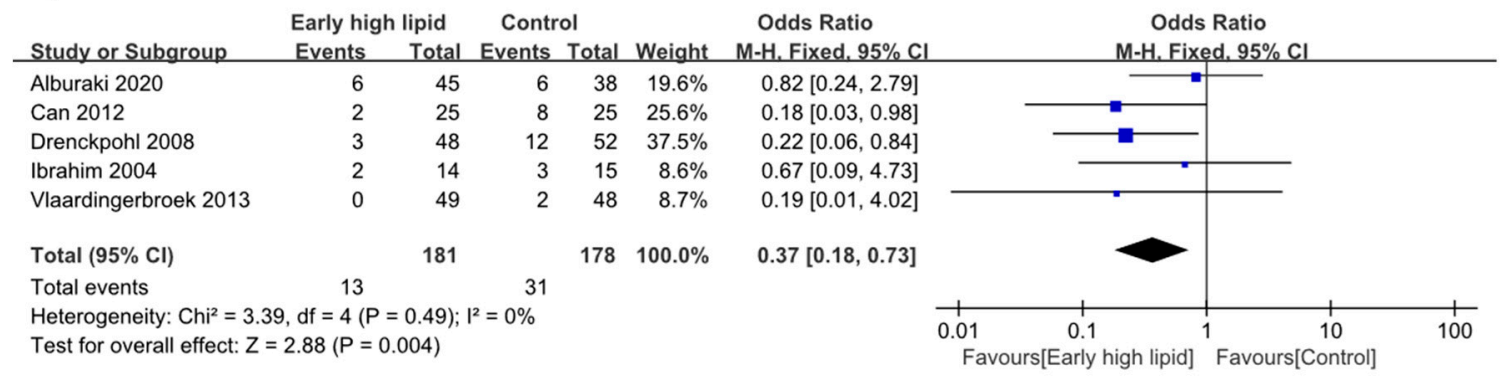

(h) Incidence of hypertriglyceridemia

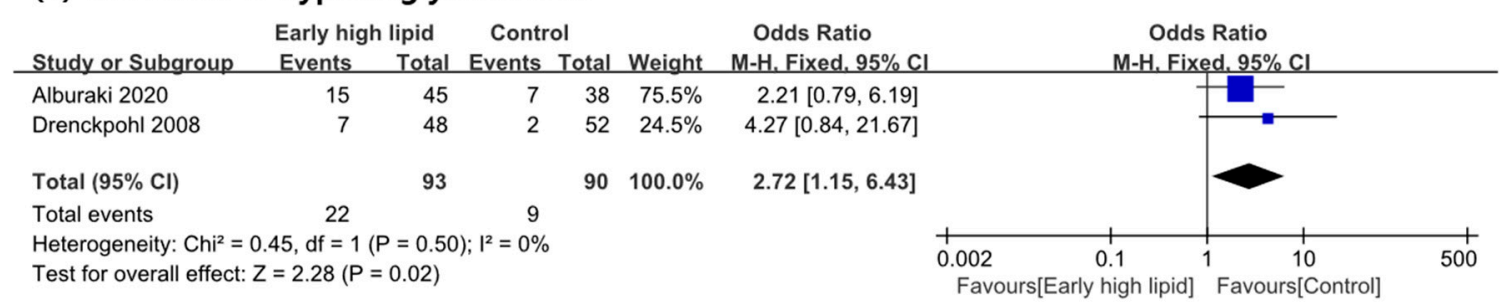

(i) Serum total bilirubin level, $\mathrm{mg} / \mathrm{dL}$

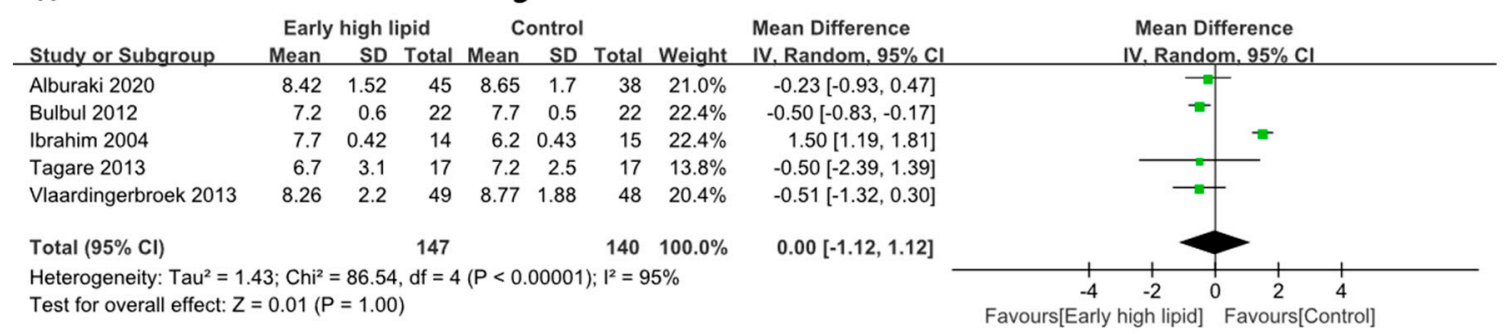

Figure 4. (a-i): Meta-analysis of the effects of early high IVLE that reached a lipid dose equal to or greater than $1.5 \mathrm{~g} / \mathrm{kg} / \mathrm{day}$ within the first $24 \mathrm{~h}$ on clinical outcomes and morbidities in preterm infants compared with controls (random effects). IV, inverse variance; $\mathrm{M}-\mathrm{H}$, Mantel-Haenszel; $\mathrm{CI}$, confidence interval; NICU, neonatal intensive care unit; BPD, bronchopulmonary dysplasia; NEC, necrotizing enterocolitis; IVH, intraventricular hemorrhage; ROP, retinopathy of prematurity.

\section{Discussion}

In this meta-analysis, the efficacy and safety of early high IVLE administration, namely, equal to or more than $1.5 \mathrm{~g} / \mathrm{kg}$ /day within the first $24 \mathrm{~h}$ of life, was evaluated through nine studies and 520 preterm infants. The results suggested several noticeable findings: early lipid initiation and increasing to high dose immediately in preterm infants seems not only better for growth but also safe. Through the intervention of early high IVLE administration, the mean postnatal weight loss decreased, the mean body weight and mean head circumference near TEA increased, and the incidence of EUGR decreased. Among the secondary outcomes, the incidence of ROP was reduced with early high lipids.

According to previous meta-analyses, the early initiation of IVLE did not result in advantages in terms of growth and did not generate a higher incidence of adverse outcomes $[4,5]$. In contrast, in the current meta-analysis, based on whether a high dose 
was achieved within $24 \mathrm{~h}$ after birth, there were advantages in terms of growth. The mean maximal percentage of postnatal weight loss was significantly lower in the treatment group, and body weight and head circumference measured near TEA were significantly higher and larger, respectively, in the early high lipid group than in the control group. The PMA at which individual studies measured anthropometrics varied; therefore, the meaning of the result should be interpreted cautiously. However, one of the most consequential findings in our meta-analysis was that the incidence of EUGR calculated considering the corrected age favored early high IVLE introduction for the growth of preterm infants; the incidence of EUGR was 0.27 times lower in the treatment group. There were two perspectives on the major differences from previous meta-analyses. First, the intervention evaluated in this meta-analysis was to assess the preservation of the physiologic intrauterine environment after birth; in other words, whether a high lipid dose (equal to or more than $1.5 \mathrm{~g} / \mathrm{kg} /$ day) was reached within $24 \mathrm{~h}$ after birth. Second, their definitions of early lipids were later than ours: a 5-day difference and 2-day difference in the initiation of lipids: in the study of Simmer et al. [4] and Vlaardingerbroek et al. [5], respectively.

During the last trimester of pregnancy, a brain growth spurt occurs in the human fetus with considerable lipid accretion [27], and appropriate lipid supplementation is crucial to preterm infants exposed to the extrauterine environment in this period. From a cohort study of mothers and infants in Ireland, AA Geraghty and colleagues reported that the serum level of total cholesterol and TG measured in cord blood at delivery were $40.71 \mathrm{mg} / \mathrm{dL}$ and $65.74 \mathrm{mg} / \mathrm{dL}$, respectively [28]. The fetus receives a substantial amount of lipids from the mother before delivery; however, these are suddenly discontinued after birth. Because of continuity with the intrauterine environment, high lipid supplementation immediately after birth is a more physiological method. Additionally, a sufficient amount of energy supplementation in the critical period might help long-term neurodevelopment and growth. Previously, Stephens et al. said that increased first-week protein and energy intakes are positively associated with higher scores in developmental tests and negatively associated with growth at 18 months [29], and Roelants et al. reported that more infants scored under datum in the control group than infants in the standard amino acid and soybean oil fat emulsion supplementation group [20].

The early high IVLE treatment did not have significant effect on secondary outcomes: mortality; admission length; and incidence of BPD, NEC, sepsis, IVH, and serum TB level. The results of our meta-analysis were in agreement with several previous metaanalyses $[4,5,30,31]$. The current meta-analysis of the effects of early high IVLE administration on episodes of hypertriglyceridemia favored the control groups. Correani et al. reported that, with close monitoring of hypertriglyceridemia and IVLE titration at TG levels $>250 \mathrm{mg} / \mathrm{dL}$, infants whose TG level measured $>250 \mathrm{mg} / \mathrm{dL}$ during early postnatal period did not have growth, disease, and neurodevelopmental disadvantages, unlike the control group [32]. Therefore, high IVLE administration appears to be safe for preterm infants with regular TG monitoring and IVLE titration.

Another interesting finding in our meta-analysis is that early high IVLE showed a favored effect on the incidence of ROP. A recently published RCT reported that enteral arachidonic acid and docosahexaenoic acid supplementation lowered the risk of severe ROP by $50 \%$ [33], which is in line with our meta-analysis results. One possible explanation of this result is that infants with early high IVLE might receive an additional source of long-chain polyunsaturated fatty acids in the early postnatal period, and fatty acids could be used for retinal development. Consequently, early high lipid administration may play a key role in reducing the incidence of ROP in preterm infants.

The objective of our meta-analysis, which was to compare "early high" lipids to a control treatment, was different from those of previous studies, and the RCTs included in the current meta-analysis were not duplicated. Simmer et al. [4] and Vlaardingerbroek et al. [5] included a total of seven RCTs [34-40]. In the study of Gilbertson et al., infants in the "early" lipid group received IVLE from $1 \mathrm{~g} / \mathrm{kg} /$ day on day 1 to $3 \mathrm{~g} / \mathrm{kg} /$ day on day 4 [39], and in the studies of Sosenko et al., infants were assigned to the "early" lipid 
group starting IVLE at less than $12 \mathrm{~h}$, at $0.5 \mathrm{~g} / \mathrm{kg} /$ day for the first $24 \mathrm{~h}$ with an increment of $0.5 \mathrm{~g} / \mathrm{kg} / 24 \mathrm{~h}$ until $1.5 \mathrm{~g} / \mathrm{kg}$ was reached [40]. In contrast, because we defined the intervention group as the "early high" lipid group that reached a lipid dose equal to or greater than $1.5 \mathrm{mg} / \mathrm{kg}$ /day within the first $24 \mathrm{~h}$, we excluded the RCTs included in previous meta-analyses. The RCT by Tan and Cooke was also excluded for the same reason [41].

The main strength of the current study was that we considered the importance of achieving a high administration dose early as well as early initiation of IVLE, which was distinctly different from previous meta-analyses. We performed a systematic review setting with only patients and interventions in a broad search. In addition, to obtain a high level of evidence, only RCTs were included. However, there were a few limitations of the current meta-analysis. First, there were concerns about the effect of the type of lipid on growth and adverse outcomes. The IVLE used in the RCTs included in our meta-analysis was soybean-based oil emulsion in large part, but Dongming et al. did not describe the exact name of lipid emulsion [17], and Vlaardingerbroek et al. used both soybean-based oil emulsions and SMOF lipid emulsion in the intervention group [19]. The second limitation of the current study was the effects of amino acids. There were differences in protein supplementation dose between early high IVLE groups and control groups in all included studies, which may have acted as a confounding factor to the effect of lipids on growth and adverse outcomes. According to ESPGHAN guidelines on parenteral nutrition [42], in preterm infants, the amino acid supply should start on the first day of life with at least $1.5 \mathrm{~g} / \mathrm{kg} /$ day to achieve an anabolic state and be increased up to between $2.5 \mathrm{~g} / \mathrm{kg} /$ day and $3.5 \mathrm{~g} / \mathrm{kg} /$ day from postnatal day 2. Seven RCTs of the included current meta-analysis satisfied current recommendations; however, in the study of Dongming et al., the infants in the control group were treated with only glucose infusion within 3 days after birth [17], resulting in nutritional deficits. In addition, the types of amino acids given to the preterm infants were different.

\section{Conclusions}

In conclusion, proactive IVLE at high doses is tolerated by preterm infants immediately after birth, and they did not show a significantly increased risk of adverse metabolic outcomes, such as hypertriglyceridemia, hypoglycemia, and hypoglycemia. In addition, there were certain beneficial effects on growth; therefore, early initiation with a high dose of IVLE should be recommended for preterm infants. Large-scale RCTs in preterm infants are prerequisite for determining the optimum dose of IVLE in the first $24 \mathrm{~h}$, the type of IVLE that should be used, and the long-term benefits of early high lipid administration. We would suggest the early initiation with a high dose of IVLE should be recommended for preterm infants.

Supplementary Materials: The following are available online at https:/ /www.mdpi.com/article/ 10.3390/nu13051535/s1, Figure S1: Subgroup meta-analysis of the effects on clinical outcome and morbidities of early high IVLE. IVLE, intravenous lipid emulsion; IV, inverse variance; $\mathrm{M}-\mathrm{H}, \mathrm{CI}$, confidence interval.

Author Contributions: Conceptualization, S.Y.K. and K.K.; methodology, S.Y.K.; validation, K.K.; formal analysis, K.K.; investigation, S.Y.K. and K.K.; resources, N.J.K.; data curation, N.J.K.; writingoriginal draft preparation, S.Y.K. and K.K.; writing-review and editing, S.Y.K. and K.K.; visualization, K.K.; supervision, S.Y.K.; project administration, S.Y.K. All authors have read and agreed to the published version of the manuscript.

Funding: This research received no external funding.

Institutional Review Board Statement: Not applicable.

Informed Consent Statement: Not applicable.

Data Availability Statement: Data is contained within the article or supplementary material. 
Conflicts of Interest: The authors declare no conflict of interest.

\section{References}

1. Lapillonne, A.; Griffin, I.J. Feeding preterm infants today for later metabolic and cardiovascular outcomes. J. Pediatr. 2013, 162, S7-S16. [CrossRef] [PubMed]

2. Embleton, N.E.; Pang, N.; Cooke, R.J. Postnatal malnutrition and growth retardation: An inevitable consequence of current recommendations in preterm infants? Pediatrics 2001, 107, 270-273. [CrossRef]

3. Rochow, N.; Raja, P.; Liu, K.; Fenton, T.; Landau-Crangle, E.; Göttler, S.; Jahn, A.; Lee, S.; Seigel, S.; Campbell, D. Physiological adjustment to postnatal growth trajectories in healthy preterm infants. Pediatr. Res. 2016, 79, 870-879. [CrossRef]

4. Simmer, K.; Rao, S.C. Early introduction of lipids to parenterally-fed preterm infants. Cochrane Database Syst. Rev. 2005, 18, CD005256.

5. Vlaardingerbroek, H.; Veldhorst, M.A.; Spronk, S.; van den Akker, C.H.; van Goudoever, J.B. Parenteral lipid administration to very-low-birth-weight infants-early introduction of lipids and use of new lipid emulsions: A systematic review and metaanalysis. Am. J. Clin. Nutr. 2012, 96, 255-268. [CrossRef] [PubMed]

6. Fischer, C.J.; Maucort-Boulch, D.; Megnier-Mbo, C.M.E.; Remontet, L.; Claris, O. Early parenteral lipids and growth velocity in extremely-low-birth-weight infants. Clin. Nutr. 2014, 33, 502-508. [CrossRef] [PubMed]

7. Dit Trolli, S.E.; Kermorvant-Duchemin, E.; Huon, C.; Bremond-Gignac, D.; Lapillonne, A. Early lipid supply and neurological development at one year in very low birth weight (VLBW) preterm infants. Early Hum. Dev. 2012, 88, S25-S29. [CrossRef]

8. Lapillonne, A.; Mis, N.F.; Goulet, O.; van den Akker, C.H.; Wu, J.; Koletzko, B.; Braegger, C.; Bronsky, J.; Cai, W.; Campoy, C.; et al. ESPGHAN/ESPEN/ESPR/CSPEN guidelines on pediatric parenteral nutrition: Lipids. Clin. Nutr. 2018, 37, 2324-2336. [CrossRef] [PubMed]

9. Alburaki, W.; Yusuf, K.; Dobry, J.; Sheinfeld, R.; Alshaikh, B. High Early Parenteral Lipid in Very Preterm Infants: A RandomizedControlled Trial. J. Pediatr. 2021, 228, 16-23. [CrossRef]

10. Moher, D.; Liberati, A.; Tetzlaff, J.; Altman, D.G.; PRISMA Group. Preferred reporting items for systematic reviews and meta-analyses: The PRISMA statement. PLoS Med. 2009, 6, e1000097. [CrossRef]

11. Fenton, T.R.; Nasser, R.; Eliasziw, M.; Kim, J.H.; Bilan, D.; Sauve, R. Validating the weight gain of preterm infants between the reference growth curve of the fetus and the term infant. BMC Pediatr. 2013, 13, 1-10. [CrossRef] [PubMed]

12. Bell, M.J.; Ternberg, J.L.; Feigin, R.D.; Keating, J.P.; Marshall, R.; Barton, L.; Brotherton, T. Neonatal necrotizing enterocolitis. Therapeutic decisions based upon clinical staging. Ann. Surg. 1978, 187, 1. [CrossRef] [PubMed]

13. Papile, L.-A.; Burstein, J.; Burstein, R.; Koffler, H. Incidence and evolution of subependymal and intraventricular hemorrhage: A study of infants with birth weights less than 1500 gm. J. Pediatr. 1978, 92, 529-534. [CrossRef]

14. International Committee for the Classification of Retinopathy of Prematurity. The international classification of retinopathy of prematurity revisited. Arch. Ophthalmol. 2005, 123, 991-999. [CrossRef] [PubMed]

15. Higgins, J.; Green, S. 17.8. 2 Study summaries using more than one patient-reported outcome. In Cochrane Handbook for Systematic Reviews of Interventions, Version 6.2; Cochrane: London, UK, 2011.

16. Higgins, J.P.; Thompson, S.G.; Deeks, J.J.; Altman, D.G. Measuring inconsistency in meta-analyses. BMJ 2003, 327, 557-560. [CrossRef]

17. Dongming, L.; Fengran, Z.; Zhaojun, Z. The study of early intravenous nutrition therapy in very low birth weight infants. Pak. J. Pharm. Sci. 2016, 29, 2293-2295.

18. Tagare, A.; Walawalkar, M.; Vaidya, U. Aggressive parenteral nutrition in sick very low birth weight babies: A randomized controlled trial. Indian Pediatr. 2013, 50, 954-956. [CrossRef]

19. Vlaardingerbroek, H.; Vermeulen, M.J.; Rook, D.; van den Akker, C.H.; Dorst, K.; Wattimena, J.L.; Vermes, A.; Schierbeek, H.; van Goudoever, J.B. Safety and efficacy of early parenteral lipid and high-dose amino acid administration to very low birth weight infants. J. Pediatr. 2013, 163, 638-644. [CrossRef]

20. Roelants, J.A.; Vlaardingerbroek, H.; van den Akker, C.H.; de Jonge, R.C.; van Goudoever, J.B.; Vermeulen, M.J. Two-Year Follow-up of a Randomized Controlled Nutrition Intervention Trial in Very Low-Birth-Weight Infants. J. Parenter. Enter. Nutr. 2018, 42, 122-131. [CrossRef]

21. Bulbul, A.; Okan, F.; Bulbul, L.; Nuhoglu, A. Effect of low versus high early parenteral nutrition on plasma amino acid profiles in very low birth-weight infants. J. Matern. Fetal Neonatal Med. 2012, 25, 770-776. [CrossRef]

22. Can, E.; Bülbül, A.; Uslu, S.; Cömert, S.; Bolat, F.; Nuhoğlu, A. Effects of aggressive parenteral nutrition on growth and clinical outcome in preterm infants. Pediatr. Int. 2012, 54, 869-874. [CrossRef] [PubMed]

23. Drenckpohl, D.; McConnell, C.; Gaffney, S.; Niehaus, M.; Macwan, K.S. Randomized trial of very low birth weight infants receiving higher rates of infusion of intravenous fat emulsions during the first week of life. Pediatrics 2008, 122, 743-751. [CrossRef] [PubMed]

24. Ibrahim, H.M.; Jeroudi, M.A.; Baier, R.; Dhanireddy, R.; Krouskop, R.W. Aggressive early total parental nutrition in low-birthweight infants. J. Perinatol. 2004, 24, 482-486. [CrossRef] [PubMed]

25. Jobe, A.H.; Bancalari, E. Bronchopulmonary dysplasia. Am. J. Respir. Crit. Care Med. 2001, 163, 1723-1729. [CrossRef]

26. Walsh, M.C.; Yao, Q.; Gettner, P.; Hale, E.; Collins, M.; Hensman, A.; Everette, R.; Peters, N.; Miller, N.; Muran, G.; et al. Impact of a physiologic definition on bronchopulmonary dysplasia rates. Pediatrics 2004, 114, 1305-1311. [CrossRef] 
27. Larqué, E.; Demmelmair, H.; Gil-Sánchez, A.; Prieto-Sánchez, M.T.; Blanco, J.E.; Pagán, A.; Faber, F.L.; Zamora, S.; Parrilla, J.J.; Koletzko, B. Placental transfer of fatty acids and fetal implications. Am. J. Clin. Nutr. 2011, 94, 1908S-1913S. [CrossRef]

28. Geraghty, A.A.; Alberdi, G.; O’Sullivan, E.J.; O’Brien, E.C.; Crosbie, B.; Twomey, P.J.; McAuliffe, F.M. Maternal and fetal blood lipid concentrations during pregnancy differ by maternal body mass index: Findings from the ROLO study. BMC Pregnancy Childbirth 2017, 17, 1-7. [CrossRef]

29. Stephens, B.E.; Walden, R.V.; Gargus, R.A.; Tucker, R.; McKinley, L.; Mance, M.; Nye, J.; Vohr, B.R. First-week protein and energy intakes are associated with 18-month developmental outcomes in extremely low birth weight infants. Pediatrics 2009, 123, 1337-1343. [CrossRef]

30. Fox, G.F.; Wilson, D.C.; Ohlsson, A. Effect of Early Vs. Late Introduction of Intravenous Lipid To Preterm Infants on Death and Chronic Lung Disease (CLD)-Results of Meta-Analysest 1250. Pediatr. Res. 1998, 43, 214. [CrossRef]

31. Wilson, D.; Fox, G.; Ohlsson, A. Meta-analyses of effects of early or late introduction of intravenous lipid to preterm infants on mortality and chronic lung disease. J. Pediatr. Gastroenterol. Nutr. 1998, 26, 599. [CrossRef]

32. Correani, A.; Giretti, I.; Antognoli, L.; Monachesi, C.; Cogo, P.; D’Ascenzo, R.; Biagetti, C.; Carnielli, V.P. Hypertriglyceridemia and Intravenous Lipid Titration During Routine Parenteral Nutrition in Small Preterm Infants. J. Pediatr. Gastroenterol. Nutr. 2019, 69, 619-625. [CrossRef]

33. Hellström, A.; Nilsson, A.K.; Wackernagel, D.; Pivodic, A.; Vanpee, M.; Sjöbom, U.; Hellgren, G.; Hallberg, B.; Domellöf, M.; Klevebro, S.; et al. Effect of Enteral Lipid Supplement on Severe Retinopathy of Prematurity: A Randomized Clinical Trial. JAMA Pediatr. 2021, 175, 359-367. [CrossRef]

34. Alwaidh, M.; Bowden, L.; Shaw, B.; Ryan, S. Randomised trial of effect of delayed intravenous lipid administration on chronic lung disease in preterm neonates. J. Pediatr. Gastroenterol. Nutr. 1996, 22, 303-306. [CrossRef] [PubMed]

35. Brownlee, K.; Kelly, E.; Ng, P.; Kendall-Smith, S.; Dear, P. Early or late parenteral nutrition for the sick preterm infant? Arch. Dis. Child. 1993, 69, 281-283. [CrossRef] [PubMed]

36. Hammerman, C.; Aramburo, M.J. Decreased lipid intake reduces morbidity in sick premature neonates. J. Pediatr. 1988, 113, 1083-1088. [CrossRef]

37. Gunn, T.; Reaman, G.; Outerbridge, E.; Colle, E. Peripheral total parenteral nutrition for premature infants with the respiratory distress syndrome: A controlled study. J. Pediatr. 1978, 92, 608-613. [CrossRef]

38. Wilson, D.C.; Cairns, P.; Halliday, H.L.; Reid, M.; McClure, G.; Dodge, J.A. Randomised controlled trial of an aggressive nutritional regimen in sick very low birthweight infants. Arch. Dis. Child. Fetal Neonatal Ed. 1997, 77, F4-F11. [CrossRef]

39. Gilbertson, N.; Kovar, I.Z.; Cox, D.J.; Crowe, L.; Palmer, N.T. Introduction of intravenous lipid administration on the first day of life in the very low birth weight neonate. J. Pediatr. 1991, 119, 615-623. [CrossRef]

40. Sosenko, I.R.; Rodriguez-Pierce, M.; Bancalari, E. Effect of early initiation of intravenous lipid administration on the incidence and severity of chronic lung disease in premature infants. J. Pediatr. 1993, 123, 975-982. [CrossRef]

41. Tan, M.J.; Cooke, R.W. Improving head growth in very preterm infants-a randomised controlled trial I: Neonatal outcomes. Arch. Dis. Child. Fetal Neonatal Ed. 2008, 93, F337-F341. [CrossRef]

42. Van Goudoever, J.B.; Carnielli, V.; Darmaun, D.; de Pipaon, M.S.; Braegger, C.; Bronsky, J.; Cai, W.; Campoy, C.; Decsi, T.; Domellöf, M.; et al. ESPGHAN/ESPEN/ESPR/CSPEN guidelines on pediatric parenteral nutrition: Amino acids. Clin. Nutr. 2018, 37, 2315-2323. [CrossRef] [PubMed] 